\title{
Self-Triggered Consensus of Vehicle Platoon System With Time-Varying Topology
}

\author{
Weiping Wang ${ }^{1,2,3,4 *}$, Chunyang Wang ${ }^{1,2,3,4}$, Yongzhen Guo ${ }^{5,6 *}$, Xiong Luo ${ }^{1,2,3,4}$ and \\ Yang $\mathrm{Gao}^{7}$
}

${ }^{1}$ School of Computer and Communication Engineering, University of Science and Technology Beijing, Beijing, China, ${ }^{2}$ Beijing Key Laboratory of Knowledge Engineering for Materials Science, Beijing, China, ${ }^{3}$ Institute of Artificial Intelligence, University of Science and Technology Beijing, Beijing, China, ${ }^{4}$ Shunde Graduate School, Beijing University of Science and Technology, Guangzhou, China, ${ }^{5}$ Industrial Control System Evaluation and Certification Department of China Software Testing Center, Beijing, China, ${ }^{6}$ School of Automation, Beijing Institute of Technology, Beijing, China, ${ }^{7}$ China Information Technology Security Evaluation Center, Beijing, China

This paper focuses on the consensus problem of a vehicle platoon system with time-varying topology via self-triggered control. Unlike traditional control methods, a more secure event-triggered controller considering the safe distance was designed for the vehicle platoon system. Then, a Lyapunov function was designed to prove the stability of the platoon system. Furthermore, based on the new event-triggered function, a more

OPEN ACCESS

Edited by:

Zijun Zhang,

City University of Hong Kong,

Hong Kong

Reviewed by:

He Chen,

Hebei University of Technology, China

Panpan Yang,

Chang'an University, China

Mingwen Zheng,

Shandong University of Technology,

China

Hui Zhao,

University of Jinan, China

*Correspondence:

Weiping Wang

weipingwangjt@ustb.edu.cn

Yongzhen Guo

yzguo@cstc.org.cn

Received: 13 April 2020

Accepted: 06 June 2020

Published: 14 October 2020

Citation:

Wang W, Wang C, Guo Y, Luo X and

Gao Y (2020) Self-Triggered

Consensus of Vehicle Platoon System

With Time-Varying Topology.

Front. Neurorobot. 14:53.

doi: 10.3389/fnbot.2020.00053 energy efficient self-triggered control strategy was designed by using the Taylor formula. The new self-triggered control strategy can directly calculate the next trigger according to the state information of the last trigger. It avoids continuous calculation and measurement of vehicles. Finally, the effectiveness of the proposed two self-triggered control strategies were verified by numerical simulation experiments.

Keywords: consense, event-triggered, self-triggered, distributed control, time-varying topology

\section{INTRODUCTION}

In recent years, multi-agent systems have been widely applied in intelligent transportation (Hee Lee et al., 2013; Vilarinho et al., 2016). As an important part of the intelligent transportation system, the self-driving vehicle platoon system has a wide range of applications in improving road utilization, enhancing safety and reliability, and alleviating traffic congestion.

The formation of control is an important issue for the vehicle platoon system. It refers to the control problem that a group of intelligent vehicles can interact with each other to maintain a predetermined geometric formation during the movement of a specific target or direction. In general, this mutual interaction between intelligent vehicles can be divided into fixed and timevarying topology. Most of the current research is mainly focused on a fixed topology (Peters et al., 2016; Viegas et al., 2018). However, in the actual driving process, the vehicle platoon system often has to face various complex terrain and traffic conditions. Formations do not stay the same all the time. The vehicle platoon system requires a change of formation. Therefore, it is necessary to study the time-varying topology of vehicle platoon system. At present, there are few research studies on vehicle platoon systems with time-varying topology. More research is focused on multiagent systems (Munz et al., 2011; Saboori and Khorasani, 2014). For example, we found that we can design more reasonable and effective control strategies by analyzing the derivatives of timevarying topological variables (Wang et al., 2018). It is thus more practical to study the time-varying topology of the vehicle platoon system than fixed topology. 
Recently, the formation consistency of the vehicle platoon system has been widely considered. It has been applied to deal with consistency of formation control problems (Ren, 2007; Stojković and Katić, 2017; Wang et al., 2017; Li et al., 2018). Bela Lantos and Gyorgy Max achieved the formation consistency of unmanned ground vehicles by using a two-trajectory nonlinear dynamic model (Lantos and Max, 2016). Peters et al. (2016) designed a way by which each follower tracks its immediate predecessor to achieve vehicle formation consistency.

Nevertheless, in the traditional vehicle platoon system consistency study, it is assumed that the vehicle platoon system has sufficient computing resources and energy supply (Fax and Murray, 2004; Lafferriere et al., 2005). The vehicle platoon system can thus carry on a continuous information exchange and a continuous control. However, such assumption is unreasonable. More often than not, the power supply and communication bandwidth of a vehicle platoon system are limited. Recently, it has been found that event-triggered control can coordinate resources among intelligent vehicles. Many scholars are thus interested in event-triggered control. As an aperiodic control mode, event-triggered control can update the controller only when needed. That is, the controller of the intelligent vehicle takes an effect when the measurement error of the vehicle platoon system exceeds a certain threshold.

Since event-triggered control can reduce the energy loss to a certain extent, many scholars apply it to consistency research (Wei et al., 2017). The author in Chu et al. (2019) proposed an unified event-triggered and distributed observer-based controller with globally asymptotic convergence rate. The consistency of vehicle platoon system is realized by the controller. A faulttolerant controller which considered the communication timedelay and event-triggered mechanism was designed to achieve the consistency of the vehicle platoon system (Fei et al., 2019).

However, in order to obtain the next trigger moment, we need to constantly obtain the state information of surrounding vehicles and calculate whether the trigger conditions are met in the distributed event-triggered control function. It is because of continuous communication and computation that an eventtriggered control strategy cannot reduce the detection loss in essence. But the self-triggered control strategy only needs to calculate the next trigger moment based on the status information of the last trigger moment. In the self-triggered strategy, data detection is no longer required between any two triggering moments. From this perspective, the self-triggered control strategy has a better performance. Authors designed a self-triggered control strategy for the second-order multi-agent system with fixed topology to ensure the consistency of the formation system (De Persis and Frasca, 2013). As far as we know, there are few research studies made on time-varying topology under self-triggering control in vehicle platoon system, and this sparked our research.

Based on the above considerations, we studied the consistency of time-varying topology for vehicle platoon system with secondorder dynamics by using distributed event-triggered control and self-triggered control strategies. The contributions of our work are three-fold:
(1) A distributed event-triggered control function considering the safe distance between vehicles was designed, and this eventtriggered control is more energy efficient than the continuous control in Fax and Murray (2004) and Lafferriere et al. (2005).

(2) Based on the Lyapunov stability analysis method, the distributed event-triggered control function under timevarying leader and time-varying topology was given. In comparison with the fixed topology in Du et al. (2017), the research of time-varying topology is more practical. Moreover, the research on time-varying leader is of more practical significance.

(3) According to (1) and (2), two distributed self-triggered control strategies were designed. In Zhang et al. (2016), Dolk et al. (2017), Wei et al. (2017), Wen et al. (2018), Chu et al. (2019), and Li Z. et al. (2019), an event-triggered control strategy was designed. Compared with these, the self-triggered control strategy further reduces the continuous detection of adjacent vehicles. Additionally, the distributed self-triggered controller is more general and practical than some existing control methods.

The rest of this paper was organized as follows. Preliminaries and the problem formulation are given in section 2 . The eventtriggered control and self-triggered control of vehicle platoon system with time-varying topology are studied in section 3 . Two numerical simulation experiments are presented in section 4 . Lastly, conclusions are drawn in section 5.

\section{PRELIMINARIES AND PROBLEM FORMULATION}

\subsection{Graph Theory}

Consider the consensus issue of multi-agent systems with timevarying topology; a communication graph is used to describe the communication topology of these agents. An undirected graph $\mathcal{G}=(\mathcal{V}, \mathcal{E}, \mathcal{A})$ consists of a finite node set $\mathcal{V}=\{1,2, \cdots, N\}$, an edge set $\mathcal{E}$, where $\mathcal{E} \subseteq \mathcal{V} \times \mathcal{V}$, and an adjacency matrix $\mathcal{A}=$ $\left[a_{i j}\right] \in \mathbb{R}^{N \times N}$. If $(j, i) \in \mathcal{E}, a_{i j}=1$, and $a_{i j}=0$ otherwise. The neighbor set of vehicle $i$ is defined as $\mathcal{N}_{i} \triangleq\{j \in \mathcal{V} \mid(j, i) \in \mathcal{E}, j \neq i\}$. The Laplacian matrix of the graph $\mathcal{G}$ is defined as $\mathcal{L}=\left[l_{i j}\right] \in$ $\mathbb{R}^{N \times N}$, where $l_{i i}=\sum_{j \neq i} a_{i j}$ and $l_{i j}=-a_{i j}$, where $i \neq j$. Moreover, we assume that there are no self-cycles, that is $a_{i i}=0$ for any $i \in \bar{N}$. The degree matrices $\mathcal{D}=\operatorname{diag}\left\{d_{1}, \cdots, d_{N}\right\}$ are diagonal matrices, whose diagonal elements are given by $d_{i}=\sum_{j=1}^{N} a_{i j}$. The Laplacian matrix associated to $\mathcal{G}$ is defined as $\mathcal{L}=\mathcal{D}-\mathcal{A}$. The set of all neighbors of node $i$ is denoted by $\mathcal{N}_{i}=\{j \in$ $\mathcal{V}:(j, i) \in \mathcal{E}\}$. The matrix $B=\operatorname{diag}\left\{b_{1}, b_{2}, \cdots, b_{N}\right\}$, where $b_{i}$ is called the adjacency coefficient between the following vehicle $i$ and the head vehicle. If the following vehicle $i$ is adjacent to the head vehicle $b_{i}=1$, otherwise $b_{i}=0$. In this paper, we define the time interval constant $h_{i j}>0$ to control the safe distance between vehicles $i$ and $j$. At the same time, we define $h_{i}>0$ to control the safe distance between vehicle $i$ and the leader vehicle.

\subsection{Definitions and Lemmas}

ASSUMPTION 2.1. It is assumed that no topology changes happen during the trigger interval. 
ASSUMPTION 2.2. It is assumed that the communication between vehicles is good, that is, there will be no communication delay and other uncertain factors.

ASSUMPTION 2.3. Suppose that at least one spanning tree exists in $\mathcal{G}$ and the node corresponding to the header is the root of the tree. The existence of the spanning tree ensures that each following vehicle can obtain the status information from the leader.

LEMMA 2.1. $2 x^{\mathrm{T}} y \leq a x^{\mathrm{T}} x+\frac{1}{a} y^{\mathrm{T}} y$, where $a>0$, and the vectors $x$ and $y$ can be any value.

LEMMA 2.2. Satur and Kharchenko (2020) suppose the matrix $A$ is a $n \times n$ real symmetric matrix, $Y$ is an $n$-dimensional real vector, and $\lambda_{\max }(A) \geq \lambda_{i}(A) \geq \lambda_{\min }(A)(i=1,2, \ldots, N)$. One has

$$
\lambda_{\max }(A)\langle Y, Y\rangle \geq\langle A Y, Y\rangle \geq \lambda_{\min }(A)\langle Y, Y\rangle .
$$

LEMMA 2.3. Li W. et al. (2019) assuming that the function $f$ satisfies Lipschitz condition, there is a non-negative constant $l \geq$ 0 that satisfies $\left\|f\left(t, x_{i}\right)-f\left(t, x_{0}\right)\right\| \leq l\left\|x_{i}-x_{0}\right\|$, or there are non-negative constants $l_{x} \geq 0, l_{v} \geq 0$ satisfies

$$
\left\|f\left(t, x_{i}, v_{i}\right)-f\left(t, x_{0}, v_{0}\right)\right\| \leq l_{x}\left\|x_{i}-x_{0}\right\|+l_{v}\left\|v_{i}-v_{0}\right\| .
$$

\subsection{Problem Formulation}

An auto-driving vehicle formation system consisted of $n$ smart cars (see Figure 1) is considered in this paper. Between the vehicles, status information can be transmitted according to certain regulations. The $h_{i} v_{0}$ in Figure 1 is the distance between the $i$ th vehicle and the leader.

In this paper, the dynamics of the leader vehicle is described as

$$
\left\{\begin{array}{l}
\dot{x_{0}}(t)=v_{0}(t) \\
\dot{v_{0}}(t)=f\left(t, x_{0}, v_{0}\right),
\end{array}\right.
$$

where $x_{0}(t), v_{0}(t) \in \mathbf{R}^{m}$ express the displacement vector and the velocity vector of the leader vehicle, and $f\left(t, x_{0}, v_{0}\right)$ is the control input of the leader vehicle. When $f=0$, the velocity of leader vehicle is constant, when $f \neq 0$ the velocity of the leader vehicle is changing.

The dynamic equation of the $i$ th follower intelligent vehicle is described as

$$
\left\{\begin{array}{l}
\dot{x}_{i}(t)=v_{i}(t) \\
\dot{v}_{i}(t)=f\left(t, x_{i}, v_{i}\right)-f\left(t, x_{0}, v_{0}\right)+u_{i}(t),
\end{array}\right.
$$

where $x_{i}(t), v_{i}(t) \in \mathbf{R}^{m}$ express the displacement vector and the speed vector of the follower intelligent vehicle $i$ respectively, and $f\left(t, x_{i}, v_{i}\right)-f\left(t, x_{0}, v_{0}\right)+u_{i}(t)$ is the control input of the $i$ th follower intelligent vehicle.

REMARK 1. The function $f(t, x, v)$ is an acceleration function known to all vehicles, and it satisfies LEMMA 2.3.

\section{MAIN RESULT}

\subsection{Distributed Event-Triggered Control of Vehicle Platoon System With Time-Varying Topology}

In order to reduce the sensor data acquisition and the energy consumption of frequent communication between vehicles, a distributed event-triggered controller is designed in this section. In the distributed event-triggered controller, each of the following vehicle has different trigger function, and its controller update is asynchronous. When the trigger condition is satisfied, the controller of the $i$ th follower vehicle is updated at $t_{k}^{i}(k=0,1,2 \cdots)$. It is satisfied $u_{i}(t)=$ $u_{i}\left(t_{k}{ }^{i}\right)$, and $\dot{u}_{i}(t)=0, \forall t \in\left[t_{k}{ }^{i}, t_{k}{ }^{i+1}\right)$. Since the topology is time-varying, the graph $\mathcal{G}$ can be treated as $\mathcal{G}(t)$. Accordingly, $\mathcal{A}, \mathcal{L}, \mathcal{D}$, and $\mathcal{B}$ become $\mathcal{A}(t), \mathcal{L}(t), \mathcal{D}(t)$, and $\mathcal{B}(t)$. In this case, Assumption 1 and Assumption 2 still hold.

\subsubsection{The Leader Vehicle Speed Is Constant}

In this section, the leader-follower consistency problem in the case of time-varying topology, which is based on the fact that the leader vehicle speed is constant, is studied, i.e., $f\left(t, x_{0}, v_{0}\right)=0$.

To make the system consistent, we set the controller of $i$ th follower vehicle:

$$
\begin{aligned}
u_{i}(t)= & -k\left[\sum_{j \in N_{i}\left(t_{k}{ }^{i}\right)} a_{i j}\left(t_{k}^{i}\right)\left(x_{i}\left(t_{k}{ }^{i}\right)-x_{j}\left(t_{k}{ }^{i}\right)-h_{i j} v_{0}\right)\right. \\
& \left.+b_{i}\left(t_{k}^{i}\right)\left(x_{i}\left(t_{k}{ }^{i}\right)-x_{0}\left(t_{k}^{i}\right)-h_{i} v_{0}\right)\right] \\
& -k r\left[\sum_{j \in N_{i}\left(t_{k}^{i}\right)} a_{i j}\left(t_{k}^{i}\right)\left(v_{i}\left(t_{k}{ }^{i}\right)-v_{j}\left(t_{k}{ }^{i}\right)\right)+b_{i}\left(t_{k}{ }^{i}\right)\left(v_{i}\left(t_{k}{ }^{i}\right)(5)\right.\right. \\
& \left.\left.-v_{0}\left(t_{k}{ }^{i}\right)\right)\right], \quad t \in\left[t_{k}^{i}, t_{k}^{i+1}\right),
\end{aligned}
$$

where $k$ and $r$ are control gains, and $N_{i}\left(t_{k}{ }^{i}\right)$ represents the set of neighbors of the $i$ th follower vehicle at $t_{k}{ }^{i}$.

In order to describe the displacement and speed tracking between the following vehicle $i$ and the leader vehicle, we defined the displacement error $\varepsilon_{i}$ and the velocity error $\eta_{i}$ as follows:

$$
\begin{aligned}
\varepsilon_{i}(t) & =x_{i}(t)-x_{0}(t)-h_{i} v_{0}, \\
\eta_{i}(t) & =v_{i}(t)-v_{0}(t) .
\end{aligned}
$$

The measurement error $e_{i}^{x}(t)$ and $e_{i}^{v}(t)$ are designed to represent the displacement and velocity differences between the triggering and the measuring moments of the $i$ th follower vehicle. We have then

$$
\begin{aligned}
& e_{i}^{x}(t)=\varepsilon_{i}\left(t_{k}^{i}\right)-\varepsilon_{i}(t), \\
& e_{i}^{v}(t)=\eta_{i}\left(t_{k}^{i}\right)-\eta_{i}(t) .
\end{aligned}
$$




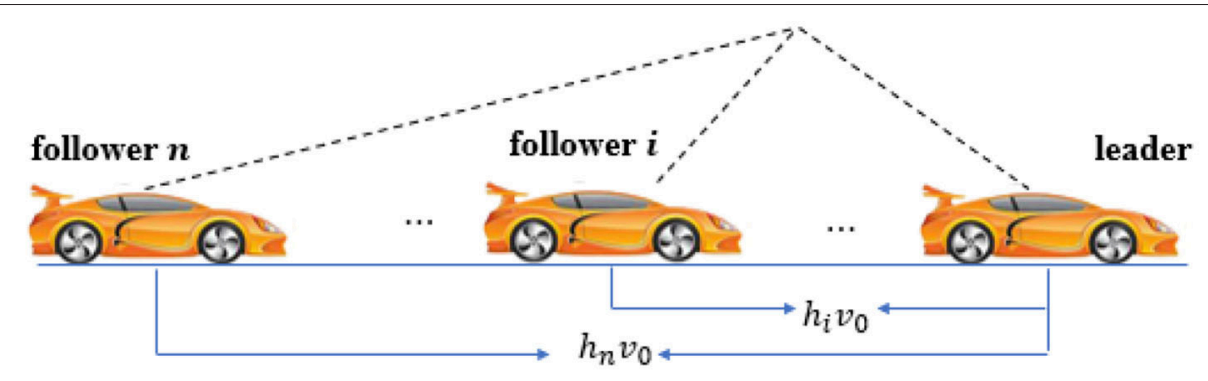

FIGURE 1 | Platoon of vehicles.

So the controller of the following intelligent vehicle becomes

$$
\begin{aligned}
u_{i}(t)= & -k\left[\sum_{j \in N_{i}(t)} a_{i j}(t)\left(\varepsilon_{i}(t)-\varepsilon_{j}(t)\right)+b_{i}(t) \varepsilon_{i}(t)\right] \\
& -k r\left[\sum_{j \in N_{i}(t)} a_{i j}(t)\left(\eta_{i}(t)-\eta_{j}(t)\right)+b_{i}(t) \eta_{i}(t)\right] \\
& -k\left[\sum_{j \in N_{i}(t)} a_{i j}(t)\left(e_{i}^{x}(t)-e_{j}^{x}(t)\right)+b_{i}(t) e_{i}^{x}(t)\right] \\
& -k r\left[\sum_{j \in N_{i}(t)} a_{i j}(t)\left(e_{i}^{v}(t)-e_{j}^{v}(t)\right)+b_{i}(t) e_{i}^{v}(t)\right] .
\end{aligned}
$$

The states and the measurement errors of intelligent vehicle are written in the vector form:

$$
\begin{aligned}
\varepsilon(t) & =\operatorname{col}\left(\varepsilon_{1}(t), \varepsilon_{2}(t), \ldots, \varepsilon_{N}(t)\right), \\
\eta(t) & =\operatorname{col}\left(\eta_{1}(t), \eta_{2}(t), \ldots, \eta_{3}(t)\right), \\
e^{x}(t) & =\operatorname{col}\left(e_{1}^{x}(t), e_{2}^{x}(t), \ldots, e_{N}^{x}(t)\right), \\
e^{v}(t) & =\operatorname{col}\left(e_{1}^{v}(t), e_{2}^{v}(t), \ldots, e_{N}^{v}(t)\right) .
\end{aligned}
$$

According to (9), we have

$$
\left\{\begin{aligned}
\dot{\varepsilon}(t)= & \eta(t) \\
\dot{\eta}(t)= & -k(L(t)+B(t)) \otimes I_{m} \varepsilon(t)-r k(L(t)+B(t)) \\
& \otimes I_{m} \eta(t)-k(L(t)+B(t)) \otimes I_{m} e^{x}(t) \\
& -r k(L(t)+B(t)) \otimes I_{m} e^{v}(t) .
\end{aligned}\right.
$$

Theorem 3.1. Consider a fleet of $N+1$ vehicles, where the dynamic equations of the head vehicle and the follower vehicle are (3) and (4). If the following conditions are met under the controller (8), then

(1) The proposed event triggering function satisfies

$$
\zeta\left(\left\|\varepsilon_{i}(t)\right\|^{2}+\left\|\eta_{i}(t)\right\|^{2}\right) \leq M\left(\left\|e_{i}^{x}(t)\right\|^{2}+\left\|e_{i}^{v}(t)\right\|^{2}\right)
$$

where $M=\frac{k a \lambda_{\min }(H(t))}{2}, H(t)=L(t)+B(t)$ and $\zeta$ will be indicated below. When this condition is met, the controller automatically updates, that is, the trigger time is reached.

(2) The minimum eigenvalue of $(L(t)+B(t)) \otimes I_{m}$ is greater than zero, which is greater than an arbitrarily small normal number $\delta$.

$$
\lambda_{\min }\left((L(t)+B(t)) \otimes I_{m}\right) \geq \delta>0 .
$$

(3) The differential coefficient of $(L(t)+B(t)) \otimes I_{m}$ exists, and the maximum eigenvalue of its derivative is greater than zero; for any small positive number, $\sigma$ is satisfied

$$
\lambda_{\max }\left(d\left((L(t)+B(t)) \otimes I_{m}\right) / d t\right) \geq \sigma>0,
$$

(4) The relation between $\eta(t)$ and $e^{x}(t), e^{v}(t)$ is

$$
\begin{aligned}
& -<k H(t) \otimes I_{m} e^{x}(t), \eta(t)>\leq-\frac{k a \lambda_{\min }(H(t))}{2}\left\|e^{x}(t)\right\| \\
& +\frac{k \lambda_{\min }(H(t))}{2 a}\|\eta(t)\|, \\
& -<k H(t) \otimes I_{m} e^{v}(t), \eta(t)>\leq-\frac{k a \lambda_{\min }(H(t))}{2}\left\|e^{v}(t)\right\| \\
& +\frac{k \lambda_{\min }(H(t))}{2 a}\|\eta(t)\|,
\end{aligned}
$$

where $\zeta=\max \left(\frac{k \psi}{2},\left(\frac{1}{a}-1\right) k \lambda_{\min }(H(t))\right), 0<a<1, \psi=$ $\lambda_{\max }\left(d\left((L(t)+B(t)) \otimes I_{m}\right) / d t\right)$, then all the vehicle reach the same state, and the existence of the safe distance $h_{i j} v_{0}$ avoid a collision. Hence, the problem of intelligent vehicle formation is solved, i.e., for $i=1,2, \ldots, N$, we have

$$
\begin{gathered}
\lim _{t \rightarrow \infty}\|\varepsilon(t)\|=0, \\
\lim _{t \rightarrow \infty}\|\eta(t)\|=0 .
\end{gathered}
$$

Proof. Based on system (10), we can construct the common Lyapunov function candidate

$$
\begin{aligned}
V(t)= & \int_{0}^{1}<k H(t) \otimes I_{m} \omega \varepsilon(t), \varepsilon(t)>d \omega \\
& +\frac{1}{2}<\eta(t), \eta(t)>
\end{aligned}
$$

where $H(t)=L(t)+B(t)$.

Firstly, we prove the positivity of $V(t)$

$$
\begin{aligned}
V & \geq \frac{1}{2} k \lambda_{\min }\left(H(t) \otimes I_{m}\right)\|\varepsilon(t)\|^{2}+\frac{1}{2}\langle\eta(t), \eta(t)\rangle \\
& =\frac{1}{2} k \lambda_{\min }\left(H(t) \otimes I_{m}\right)\|\varepsilon(t)\|^{2}+\frac{1}{2}\|\eta(t)\|^{2} \\
& \geq \frac{1}{2} \min \{k \zeta, 1\}\left(\|\varepsilon(t)\|^{2}+\|\eta(t)\|^{2}\right) .
\end{aligned}
$$


It can be seen that the Lyapunov function (15) selected is positively definite.

The time derivative of (15) can be expressed as

$$
\begin{aligned}
\frac{\mathrm{d} V}{\mathrm{~d} t}= & \frac{\mathrm{d}}{\mathrm{d} t} \int_{0}^{1}<k H(t) \otimes I_{m} \omega \varepsilon(t), \varepsilon(t)>d \omega \\
& -<k H(t) \otimes I_{m} \varepsilon(t), \eta(t)> \\
& -<k H(t) \otimes I_{m} \eta(t), \eta(t)> \\
& -<k H(t) \otimes I_{m} e^{x}(t), \eta(t)> \\
& -<k H(t) \otimes I_{m} e^{v}(t), \eta(t)>.
\end{aligned}
$$

Taking out the first term, we have

$$
\begin{aligned}
\frac{\mathrm{d}}{\mathrm{d} t} \int_{0}^{1}< & k H(t) \otimes I_{m} \omega \varepsilon(t), \varepsilon(t)>d \omega \\
= & \int_{0}^{1}<k H(t) \otimes I_{m} \omega \eta(t), \varepsilon(t)>d \omega \\
+ & \int_{0}^{1}<k H(t) \otimes I_{m} \omega \varepsilon(t), \eta(t)>d \omega \\
& +\int_{0}^{1}<k \frac{\mathrm{d}(H(t))}{\mathrm{d} t} \otimes I_{m} \omega \varepsilon(t), \varepsilon(t)>d \omega \\
= & <k H(t) \otimes I_{m} \varepsilon(t), \eta(t)> \\
& +\int_{0}^{1}<k \frac{\mathrm{d}(H(t))}{\mathrm{d} t} \otimes I_{m} \omega \varepsilon(t), \varepsilon(t)>d \omega .
\end{aligned}
$$

By (18), we get

$$
\begin{aligned}
\frac{\mathrm{d} V}{\mathrm{~d} t}= & \int_{0}^{1}<k \frac{\mathrm{d}\left(\mathrm{H}(\mathrm{t}) \otimes \mathrm{I}_{\mathrm{m}}\right)}{\mathrm{d} t} \omega \varepsilon(t), \varepsilon(t)>d \omega-<k H(t) \\
& \otimes I_{m} \eta(t), \eta(t)>-<k H(t) \otimes I_{m} e^{x}(t), \eta(t)> \\
& -<k H(t) \otimes I_{m} e^{v}(t), \eta(t)>.
\end{aligned}
$$

Take (19) into consideration, we have

$$
\begin{aligned}
\frac{\mathrm{d} V}{\mathrm{~d} t}= & \int_{0}^{1}<k \frac{\mathrm{d}\left(H(t) \otimes I_{m}\right)}{\mathrm{d} t} \omega \varepsilon(t), \varepsilon(t)>d \omega \\
& -<k H(t) \otimes I_{m} \eta(t), \eta(t)> \\
& -\frac{k a \lambda_{\min }(H(t))}{2}\left\|e^{x}(t)\right\|^{2}+\frac{k \lambda_{\min }(H(t))}{2 a}\|\eta(t)\|^{2} \\
& -\frac{k a \lambda_{\min }(H(t))}{2}\left\|e^{v}(t)\right\|^{2}+\frac{k \lambda_{\min }(H(t))}{2 a}\|\eta(t)\|^{2} \\
\leq & \left(\frac{k \psi}{2}\|\varepsilon(t)\|^{2}+\left(\frac{1}{a}-1\right) k \lambda_{\min }(H(t))\|\eta(t)\|^{2}\right) \\
& -\left(\frac{k a \lambda_{\min }(H(t))}{2}\left(\left\|e^{x}(t)\right\|^{2}+\left\|e^{v}(t)\right\|^{2}\right)\right) \\
\leq & s\left(\|\varepsilon(t)\|^{2}+\|\eta(t)\|^{2}\right) \\
& -\left(\frac{k a \lambda_{\min }(H(t))}{2}\left(\left\|e^{x}(t)\right\|^{2}+\left\|e^{v}(t)\right\|^{2}\right)\right),
\end{aligned}
$$

where $\zeta=\max \left(\frac{k \psi}{2},\left(\frac{1}{a}-1\right) k \lambda_{\min }(H(t))\right), \psi=\lambda_{\max }(\mathrm{d}((L(t)+$ $\left.\left.B(t)) \otimes I_{m}\right) / \mathrm{d} t\right)$. According to the trigger condition (11), the derivative of the Lyapunov function is less or equal to 0 , so the stability is proved.

\subsubsection{The Speed of the Leading Vehicle Is Time Varying}

In the actual situation, the speed of the leading vehicle cannot be fixed, most of them are time varying. Therefore, in this section, we study the consistency of leader followers in the case of time-varying topology based on the fact that the speed of the leader vehicle is time varying, i.e., $f\left(t, x_{0}, v_{0}\right) \neq 0$. In the meantime, suppose $f\left(t, \varepsilon_{i}(t), \eta_{i}(t)\right)=f\left(t_{k}^{i}, x_{i}\left(t_{k}^{i}\right), v_{i}\left(t_{k}{ }^{i}\right)\right)-$ $f\left(t, x_{0}\left(t_{k}^{i}\right), v_{0}\left(t_{k}^{i}\right)\right)$.

To make the system consistent, we set the $i$ th follower vehicle's controller as

$$
\begin{gathered}
u_{i}(t)=-k\left[\sum_{j \in N_{i}\left(t_{k}{ }^{i}\right)} a_{i j}\left(t_{k}^{i}\right)\left(x_{i}\left(t_{k}^{i}\right)-x_{j}\left(t_{k}{ }^{i}\right)-h_{i j} v_{0}\right)\right. \\
-k r\left[\sum_{j \in N_{i}\left(t_{k}{ }^{i}\right)} a_{i j}\left(t_{k}{ }^{i}\right)\left(v_{i}\left(t_{k}{ }^{i}\right)-t_{j}\left(t_{k}{ }^{i}\right)\right)+b_{i}\left(t_{k}^{i}\right)\left(v_{i}(t)\right.\right. \\
\left.\left.\quad-v_{0}\left(t_{k}{ }^{i}\right)\right)\right], \quad t \in\left[t_{k}^{i}, t_{k}^{i+1}\right) .
\end{gathered}
$$

Similar to (6)-(9), we can format the system (4) as follows

$$
\begin{aligned}
\dot{\varepsilon}(t)= & \eta(t) \\
\dot{\eta}(t)= & f\left(t, \varepsilon(t), \eta(t), e^{x}(t), e^{v}(t)\right)-k(L(t)+B(t)) \otimes I_{m} \varepsilon(t) \\
& -r k(L(t)+B(t)) \otimes I_{m} \eta(t)-k(L(t)+B(t)) \otimes I_{m} e^{x}(t) \\
& -r k(L(t)+B(t)) \otimes I_{m} e^{v}(t)
\end{aligned}
$$

Theorem 3.2. Consider a fleet of $N+1$ vehicles, where the dynamic equations of the head vehicle and the follower vehicle are (3) and (4) respectively. If the following conditions are met under the controller (21), then

(1) The designed event triggering function satisfies the following conditions.

$$
\begin{aligned}
\zeta\left(\left\|\varepsilon_{i}(t)\right\|^{2}+\left\|\eta_{i}(t)\right\|^{2}\right) & \leq\left(\frac{k a \lambda_{\min }(H(t))}{2}-\frac{l}{2 a_{1}}\right) \\
& \left.\times\left(\left\|e_{i}^{x}(t)\right\|^{2}+\left\|e_{i}^{v}(t)\right\|^{2}\right)\right),
\end{aligned}
$$

where $H(t)=L(t)+B(t)$ and $\zeta$ will be indicated below. When this condition is met, the controller automatically updates, that is, the trigger time is reached.

(2) The minimum eigenvalue of $(L(t)+B(t)) \otimes I_{m}$ is greater than zero. There is thus a small positive number $\delta$ satisfying

$$
\lambda_{\min }\left((L(t)+B(t)) \otimes I_{m}\right) \geq \delta>0
$$

(3) The differential coefficient of $(L(t)+B(t)) \otimes I_{m}$ exists, and the maximum eigenvalue of its derivative is greater than zero, so there exists a small positive number $\sigma$ satisfying

$$
\lambda_{\max }\left(d\left((L(t)+B(t)) \otimes I_{m}\right) / d t\right) \geq \sigma>0 .
$$


(4) The relation between $\eta(t)$ and $e^{x}(t), e^{v}(t)$ is

$$
\begin{aligned}
-<k H(t) \otimes I_{m} e^{x}(t), \eta(t)>\leq- & \frac{k a \lambda_{\min }(H(t))}{2}\left\|e^{x}(t)\right\| \\
& +\frac{k \lambda_{\min }(H(t))}{2 a}\|\eta(t)\| \\
-<k H(t) \otimes I_{m} e^{v}(t), \eta(t)>\leq- & \frac{k a \lambda_{\min }(H(t))}{2}\left\|e^{v}(t)\right\| \\
& +\frac{k \lambda_{\min }(H(t))}{2 a}\|\eta(t)\| .
\end{aligned}
$$

(5)

$$
\frac{k a \lambda_{\min }(H(t))}{2}-\frac{l}{2 a_{1}}>0
$$

where $\zeta=\max \left(\frac{k \psi}{2}+\frac{l}{2 a_{1}}, \frac{3 a_{1} l}{2}+l+\frac{k \lambda_{\min }(H(t))}{a}\right)$, $\psi=\lambda_{\max }\left(d\left((L(t)+B(t)) \otimes I_{m}\right) / d t\right), 0<a, 0<a_{1}$, $0<k$, then all the vehicles reach the same state, and at the same time the existence of safe distance $h_{i j} v_{0}$ avoid a collision. The problem of intelligent vehicle formation has been solved, i.e., for $i=1,2, \ldots, N$, we have

$$
\begin{aligned}
& \lim _{t \rightarrow \infty}\|\varepsilon(t)\|=0 \\
& \lim _{t \rightarrow \infty}\|\eta(t)\|=0
\end{aligned}
$$

PROOF. Based on system (22), we can construct the Lyapunov function candidate

$V(t)=\int_{0}^{1}<k H(t) \otimes I_{m} \omega \varepsilon(t), \varepsilon(t)>d \omega+\frac{1}{2}<\eta(t), \eta(t)>$,

where $H(t)=L(t)+B(t)$.

It can be seen that the (28) selected is positively definite.

The time derivative of (28) can be expressed as

$$
\begin{aligned}
\frac{\mathrm{d} V}{\mathrm{~d} t}= & \frac{\mathrm{d}}{\mathrm{d} t} \int_{0}^{1}<k H(t) \otimes I_{m} \omega \varepsilon(t), \varepsilon(t)>d \omega \\
& +<\eta(t), f\left(t, \varepsilon(t), \eta(t), e^{x}(t), e^{v}(t)\right)> \\
& -<k H(t) \otimes I_{m} \varepsilon(t), \eta(t)>-<k H(t) \otimes I_{m} \eta(t), \eta(t)> \\
& -<k H(t) \otimes I_{m} e^{x}(t), \eta(t)>-<k H(t) \otimes I_{m} e^{v}(t), \eta(t)>
\end{aligned}
$$

Taking out of the first term, we have

$$
\begin{aligned}
& \frac{\mathrm{d}}{\mathrm{d} t} \int_{0}^{1}<k H(t) \otimes I_{m} \omega \varepsilon(t), \varepsilon(t)>d \omega \\
= & \int_{0}^{1}<k H(t) \otimes I_{m} \omega \eta(t), \varepsilon(t)>d \omega \\
& +\int_{0}^{1}<k H(t) \otimes I_{m} \omega \varepsilon(t), \eta(t)>d \omega \\
& +\int_{0}^{1}<k \frac{\mathrm{d}(H(t))}{\mathrm{d} t} \otimes I_{m} \omega \varepsilon(t), \varepsilon(t)>d \omega \\
= & <k H(t) \otimes I_{m} \varepsilon(t), \eta(t)> \\
& +\int_{0}^{1}<k \frac{\mathrm{d}\left(H(t) \otimes I_{m}\right)}{\mathrm{d} t} \omega \varepsilon(t), \varepsilon(t)>d \omega .
\end{aligned}
$$

Using Lemma 2 to enlarge the second item in (29), we yield

$$
\begin{aligned}
<\eta(t), & f\left(t, \varepsilon(t), \eta(t), e^{x}(t), e^{v}(t)\right)> \\
& \leq\|\eta(t)\| l(\|\varepsilon(t)\|+\|\eta(t)\| \\
& \left.+\left\|e^{x}(t)\right\|+\left\|e^{v}(t)\right\|\right) .
\end{aligned}
$$

Considering (30) and (31), we get

$$
\begin{aligned}
\frac{\mathrm{d} V}{\mathrm{~d} t} \leq & \int_{0}^{1}<k \frac{\mathrm{d}\left(H(t) \otimes I_{m}\right)}{d t} \omega \varepsilon(t), \varepsilon(t)>d \omega \\
& +\|\eta(t)\| l\left(\|\varepsilon(t)\|+\|\eta(t)\|+\left\|e^{x}(t)\right\|+\left\|e^{v}(t)\right\|\right) \\
& -<k H(t) \otimes I_{m} \eta(t), \eta(t)> \\
& -<k H(t) \otimes I_{m} e^{x}(t), \eta(t)>-<k H(t) \otimes I_{m} e^{v}(t), \eta(t)>.
\end{aligned}
$$

By using Lemma 3, the above items are amplified, and then

$$
\begin{aligned}
& \|\eta(t)\| l\left(\|\varepsilon(t)\|+\|\eta(t)\|+\left\|e^{x}(t)\right\|+\left\|e^{v}(t)\right\|\right) \\
& =l(\|\eta(t)\|\|\varepsilon(t)\|+\|\eta(t)\|\|\eta(t)\| \\
& \left.+\|\eta(t)\|\left\|e^{x}(t)\right\|+\|\eta(t)\|\left\|e^{v}(t)\right\|\right), \\
& \|\eta(t)\|\|\varepsilon(t)\| \leq \frac{a_{1}}{2}\|\eta(t)\|^{2}+\frac{1}{2 a_{1}}\|\varepsilon(t)\|^{2}, \\
& \|\eta(t)\|\left\|e^{x}(t)\right\| \leq \frac{a_{1}}{2}\|\eta(t)\|^{2}+\frac{1}{2 a_{1}}\left\|e^{x}(t)\right\|^{2}, \\
& \|\eta(t)\|\left\|e^{v}(t)\right\| \leq \frac{a_{1}}{2}\|\eta(t)\|^{2}+\frac{1}{2 a_{1}}\left\|e^{v}(t)\right\|^{2},
\end{aligned}
$$

where $a_{1}>0$.

Considering (34), we have

$$
\begin{aligned}
\frac{\mathrm{d} V}{\mathrm{~d} t} \leq & \frac{k \psi}{2}\|\varepsilon(t)\|^{2}+\left(\frac{a_{1} l}{2}+l\right)\|\eta(t)\|^{2} \\
& +\frac{l}{2 a_{1}}\|\varepsilon(t)\|^{2}+\frac{a_{1} l}{2}\|\eta(t)\|^{2}+\frac{l}{2 a_{1}}\left\|e^{x}(t)\right\|^{2} \\
& +\frac{a_{1} l}{2}\|\eta(t)\|^{2}+\frac{l}{2 a_{1}}\left\|e^{v}(t)\right\|^{2}-\frac{k a \lambda_{\min }(H(t))}{2}\left\|e^{x}(t)\right\| \\
& +\frac{k \lambda_{\min }(H(t))}{2 a}\|\eta(t)\| \\
& -\frac{k a \lambda_{\min }(H(t))}{2}\left\|e^{v}(t)\right\|+\frac{k \lambda_{\min }(H(t))}{2 a}\|\eta(t)\| \\
\leq & \left(\frac{k \psi}{2}+\frac{l}{2 a_{1}}\right)\|\varepsilon(t)\|^{2}+\left(\frac{3 a_{1} l}{2}+l+\frac{k \lambda_{\min }(H(t))}{2 a}\right. \\
& \left.+\frac{k \lambda_{\min }(H(t))}{2 a}\right)\|\eta(t)\|^{2} \\
& -\left(\frac{k a \lambda_{\min }(H(t))}{2}-\frac{l}{2 a_{1}}\right)\left(\left\|e^{x}(t)\right\|^{2}+\left\|e^{v}(t)\right\|^{2}\right) \\
\leq & S\left(\|\varepsilon(t)\|^{2}+\|\eta(t)\|^{2}\right)-\left(\frac{k a \lambda_{\min }(H(t))}{2}-\frac{l}{2 a_{1}}\right) \\
& \left(\left\|e^{x}(t)\right\|^{2}+\left\|e^{v}(t)\right\|^{2}\right) .
\end{aligned}
$$

According to the trigger condition (23), the derivative of the Lyapunov function (29) is less or equal to 0, and it is constant, so the stability is proved. 


\subsection{Distributed Self-Triggered Control of Vehicle Platoon System With Time-Varying Topology}

As can be seen from the distributed event-triggered control (11) and (23), the control method reduces the dependence on the global state information and the real-time state of measurement error in the trigger interval. However, it will increase the energy consumption of the sensor and microprocessor in the process of continuous measurement error detection. In order to improve this problem, we apply the self-triggered control strategy to solve the problem of intelligent vehicle formation. Under this strategy, the next trigger moment $t_{\mathrm{k}+1}^{i}$ of the $i$ th follower vehicle can obtained according to the state of the $i$ th vehicle at the previous trigger time.

\subsubsection{The Leader Vehicle Speed Is Constant}

In this part, we will transform the event-triggered control (11) into a self-triggered control strategy for the case that the vehicle speed of the leader is constant.

We know that from the previous distributed event triggering control $\left(\left\|\varepsilon_{i}(t)\right\|^{2}+\left\|\eta_{i}(t)\right\|^{2}\right) \leq \gamma\left(\left\|e_{i}^{x}(t)\right\|^{2}+\left\|e_{i}^{v}(t)\right\|^{2}\right)$, where $\gamma=\frac{k a \lambda_{\min }(H(t))}{2 \zeta}$. Using Taylor's formula, expand $\varepsilon_{i}, \eta_{i}, e_{i}^{x}, e_{i}^{v}$ at $t_{k}^{i}$, we have

$$
\begin{aligned}
& \varepsilon_{i}(t)=\eta_{i}\left(t_{k}^{i}\right)\left(t-t_{k}^{i}\right)+\varepsilon_{i}\left(t_{k}{ }^{i}\right), \\
& \eta_{i}(t)=\dot{\eta}_{i}\left(t_{k}{ }^{i}\right)\left(t-t_{k}{ }^{i}\right)+\eta_{i}\left(t_{k}{ }^{i}\right) \\
& =\left(-k \sum_{j=1}^{N}\left(L_{j,},\left(t_{k}^{i}\right)+B_{j,}\left(t_{k}^{i}\right)\right) \varepsilon_{j}\left(t_{k}^{i}\right)\right. \\
& -k r\left(\sum_{j=1}^{N}\left(L_{j, .}\left(t_{k}^{i}\right)+B_{j,},\left(t_{k}{ }^{i}\right)\right) \eta_{j}\left(t_{k}^{i}\right)\left(t-t_{k}^{i}\right)\right) \\
& +\eta_{i}\left(t_{k}^{i}\right), \\
& e_{i}{ }^{x}=\varepsilon_{i}\left(t_{k}{ }^{i}\right)-\varepsilon_{i}(t)=-\eta_{i}\left(t_{k}{ }^{i}\right)\left(t-t_{k}{ }^{i}\right) \\
& e_{i}^{v}=\eta_{i}\left(t_{k}{ }^{i}\right)-\eta_{i}(t)=-\eta_{i}\left(t_{k}^{i}\right)\left(t-t_{k}{ }^{i}\right) \\
& =-\left(k \sum_{j=1}^{N}\left(L_{j,},\left(t_{k}^{i}\right)+B_{j, .}\left(t_{k}^{i}\right)\right) \varepsilon_{j}\left(t_{k}{ }^{i}\right)\right. \\
& \left.-k r \sum_{j=1}^{N}\left(L_{j,},\left(t_{k}^{i}\right)+B_{j,}\left(t_{k}^{i}\right)\right) \eta_{j}\left(t_{k}^{i}\right)\right)\left(t-t_{k}^{i}\right),
\end{aligned}
$$

where $L_{j}$, represents $L_{j, k}$, and $k=0,1,2, \cdots, N$.

According to the above expressions and the distributed eventtriggering control function (11), we get

$$
\begin{gathered}
\left(\left\|\eta_{i}\left(t_{k}^{i}\right)\left(t-t_{k}^{i}\right)+\varepsilon_{i}\left(t_{k}^{i}\right)\right\|^{2}+\|\right. \\
\left(k \sum_{j=1}^{N}\left(L_{j, \cdot}\left(t_{k}^{i}\right)+B_{j,}\left(t_{k}^{i}\right)\right) \varepsilon_{j}\left(t_{k}^{i}\right)\right. \\
\left.\left.-k r \sum_{j=1}^{N}\left(L_{j, .}\left(t_{k}^{i}\right)+B_{j, \cdot}\left(t_{k}^{i}\right)\right) \eta_{j}\left(t_{k}^{i}\right)\right)\left(t-t_{k}^{i}\right)+\eta_{i}\left(t_{k}^{i}\right) \|^{2}\right) \\
\leq \gamma\left(\left\|-\eta_{i}\left(t_{k}^{i}\right)\left(t-t_{k}^{i}\right)\right\|^{2}+\|\right.
\end{gathered}
$$

$$
\begin{gathered}
-\left(-k \sum_{j=1}^{N}\left(L_{j, \cdot}\left(t_{k}^{i}\right)+B_{j, .}\left(t_{k}^{i}\right)\right) \varepsilon_{j}\left(t_{k}^{i}\right)\right. \\
\left.\left.\left.-k r \sum_{j=1}^{N}\left(L_{j, .}\left(t_{k}^{i}\right)+B_{j,}\left(t_{k}{ }^{i}\right)\right) \eta_{j}\left(t_{k}^{i}\right)\right)\right)\left(t-t_{k}^{i}\right) \|^{2}\right) .
\end{gathered}
$$

In order to simplify (37), we define

$$
\Omega=\left\|\begin{array}{l}
-\left(-k \sum_{j=1}^{N}\left(L_{j, .}\left(t_{k}{ }^{i}\right)+B_{j,}\left(t_{k}{ }^{i}\right)\right) \varepsilon_{j}\left(t_{k}{ }^{i}\right)\right. \\
\left.-k r \sum_{j=1}^{N}\left(L_{j, .}\left(t_{k}^{i}\right)+B_{j,}\left(t_{k}{ }^{i}\right)\right) \eta_{j}\left(t_{k}{ }^{i}\right)\right)
\end{array}\right\|^{2}+\left\|-\eta_{i}\left(t_{k}^{i}\right)\right\|^{2},
$$

and

$$
\begin{aligned}
\pi= & -k\left(\sum_{j=1}^{N}\left(L_{j,}\left(t_{k}^{i}\right)+B_{j,}\left(t_{k}^{i}\right)\right) \varepsilon_{j}\left(t_{k}{ }^{i}\right)\right) \\
& -k r\left(\sum_{j=1}^{N}\left(L_{j,}\left(t_{k}^{i}\right)+B_{j,}\left(t_{k}^{i}\right)\right) \eta_{j}\left(t_{k}^{i}\right)\right) .
\end{aligned}
$$

Suppose $\sigma_{i}=t-t_{k}^{i}$, we have

$$
\left\|\eta_{i}\left(t_{k}{ }^{i}\right) \sigma_{i}+\varepsilon_{i}\left(t_{k}{ }^{i}\right)\right\|^{2}+\left\|\pi \sigma_{i}+\eta_{i}\left(t_{k}^{i}\right)\right\|^{2} \leq \gamma \Omega \sigma_{i}^{2} .
$$

We can see that when $\sigma_{i}=0$, the inequality is not true. So $\sigma_{i}>0$, that is to say $t-t_{k}{ }^{i}>0$. To sum up, the self-triggering control strategy of the follower vehicle at $t_{k}^{i+1}$ moment is determined by the following conditions

$$
\left\|\eta_{i}\left(t_{k}^{i}\right) \sigma_{i}+\varepsilon_{i}\left(t_{k}^{i}\right)\right\|^{2}+\left\|\sigma_{i}+\eta_{i}\left(t_{k}^{i}\right)\right\|^{2}=\gamma \Omega \sigma_{i}^{2} .
$$

$\sigma_{i}>0$ which satisfies (41), we get the next trigger time $t_{k}^{i+1}=$ $\sigma_{i}+t_{k}^{i}$. In particular, if the topology of the vehicle queue changes at time $t$, so that $t_{k}^{i+1}=t$.

REMARK 2. The existence of $\sigma_{i}$ indicates that a Zeno behavior does not exist. At the same time, it indicates that the self-triggered control strategy can realize the leader-follower consistency of vehicle formation under the condition of time-varying topology and the leader vehicle speed being the same. The proof of stability is same to event-triggered control, so we are omitted here.

\subsubsection{The Speed of the Leading Vehicle Is Time Varying}

In this part, we will transform the event-triggered control (23) into a self-triggered control strategy for the case that the vehicle speed of the leader is time varying.

We know that from the previous distributed event triggering control. $\left(\left\|\varepsilon_{i}(t)\right\|^{2}+\left\|\eta_{i}(t)\right\|^{2}\right) \leq \gamma\left(\left\|e_{i}^{x}(t)\right\|^{2}+\left\|e_{i}^{\nu}(t)\right\|^{2}\right)$ where $\gamma=\frac{k a \lambda_{\min }(H(t))}{2 \zeta}-\frac{1}{2 a_{1} \zeta}$. 
Using Taylor's formula, expand $\varepsilon_{i}, \eta_{i}, e_{i}^{x}, e_{i}^{v}$ at $t_{k}^{i}$, we have

$$
\begin{aligned}
\varepsilon_{i}(t)= & \eta_{i}\left(t_{k}^{i}\right)\left(t-t_{k}^{i}\right)+\varepsilon_{i}\left(t_{k}^{i}\right), \\
\eta_{i}(t)= & \dot{\eta}_{i}\left(t_{k}^{i}\right)\left(t-t_{k}^{i}\right)+\eta_{i}\left(t_{k}^{i}\right) \\
= & \left(f\left(t, \varepsilon\left(t_{k}^{i}\right), \eta\left(t_{k}^{i}\right), e^{x}\left(t_{k}^{i}\right), e^{v}\left(t_{k}^{i}\right)\right)\right. \\
& -k \sum_{j=1}^{N}\left(L_{j, .}\left(t_{k}^{i}\right)+B_{j, \cdot}\left(t_{k}^{i}\right)\right) \varepsilon_{j}\left(t_{k}^{i}\right) \\
& \left.-k r \sum_{j=1}^{N}\left(L_{j,}\left(t_{k}^{i}\right)+B_{j, .}\left(t_{k}^{i}\right)\right) \eta_{j}\left(t_{k}^{i}\right)\right)\left(t-t_{k}^{i}\right)+\eta_{i}\left(t_{k}^{i}\right)
\end{aligned}
$$

and

$$
\begin{aligned}
e_{i}^{x}= & \varepsilon_{i}\left(t_{k}^{i}\right)-\varepsilon_{i}(t)=-\eta_{i}\left(t_{k}^{i}\right)\left(t-t_{k}^{i}\right) \\
e_{i}^{v}= & \eta_{i}\left(t_{k}^{i}\right)-\eta_{i}(t)=-\dot{\eta}_{i}\left(t_{k}^{i}\right)\left(t-t_{k}^{i}\right) \\
= & -\left(f\left(t, \varepsilon\left(t_{k}^{i}\right), \eta\left(t_{k}^{i}\right), e^{x}\left(t_{k}^{i}\right), e^{v}\left(t_{k}^{i}\right)\right)\right. \\
& -k \sum_{j=1}^{N}\left(L_{j,}\left(t_{k}^{i}\right)+B_{j,}\left(t_{k}^{i}\right)\right) \varepsilon_{j}\left(t_{k}^{i}\right) \\
& \left.-k r \sum_{j=1}^{N}\left(L_{j,}\left(t_{k}^{i}\right)+B_{j,}\left(t_{k}^{i}\right)\right) \eta_{j}\left(t_{k}^{i}\right)\right)\left(t-t_{k}^{i}\right) .
\end{aligned}
$$

According to the above two formulas and (23), we get

$$
\begin{aligned}
& \left(\left\|\eta_{i}\left(t_{k}{ }^{i}\right)\left(t-t_{k}{ }^{i}\right)+\varepsilon_{i}\left(t_{k}^{i}\right)\right\|^{2}\right. \\
& \|\left(f \left(t, \varepsilon\left(t_{k}^{i}\right), \eta\left(t_{k}^{i}\right), e^{x}\left(t_{k}^{i}\right), e^{v}\left(t_{k}^{i}\right)\right.\right.
\end{aligned}
$$

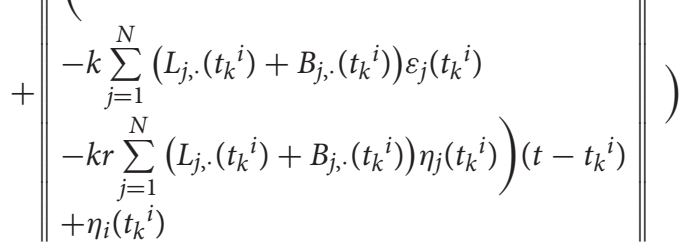

$$
\begin{aligned}
& \leq \gamma\left(\left\|-\eta_{i}\left(t_{k}^{i}\right)\left(t-t_{k}{ }^{i}\right)\right\|^{2}\right. \\
& \left.+\| \begin{array}{l}
-\left(f \left(t, \varepsilon\left(t_{k}^{i}\right), \eta\left(t_{k}^{i}\right), e^{x}\left(t_{k}^{i}\right), e^{v}\left(t_{k}^{i}\right)\right.\right. \\
-k \sum_{j=1}^{N}\left(L_{j, .}\left(t_{k}^{i}\right)+B_{j,}\left(t_{k}^{i}\right)\right) \varepsilon_{j}\left(t_{k}^{i}\right) \\
\left.-k r \sum_{j=1}^{N}\left(L_{j, .}\left(t_{k}^{i}\right)+B_{j,}\left(t_{k}^{i}\right)\right) \eta_{j}\left(t_{k}^{i}\right)\right)\left(t-t_{k}^{i}\right)
\end{array}\right) .
\end{aligned}
$$

In order to simplify (44), we define

$$
\Omega=\left\|\begin{array}{l}
-\left(f \left(t, \varepsilon\left(t_{k}^{i}\right), \eta\left(t_{k}^{i}\right), e^{x}\left(t_{k}^{i}\right), e^{v}\left(t_{k}^{i}\right)\right.\right. \\
-k \sum_{j=1}^{N}\left(L_{j,}\left(t_{k}^{i}\right)+B_{j,}\left(t_{k}{ }^{i}\right)\right) \varepsilon_{j}\left(t_{k}^{i}\right) \\
\left.-k r \sum_{j=1}^{N}\left(L_{j,}\left(t_{k}^{i}\right)+B_{j,}\left(t_{k}^{i}\right)\right) \eta_{j}\left(t_{k}^{i}\right)\right)
\end{array}\right\|^{2}+\left\|-\eta_{i}\left(t_{k}^{i}\right)\right\|^{2}
$$

$$
\begin{aligned}
\pi= & f\left(t, \varepsilon\left(t_{k}^{i}\right), \eta\left(t_{k}^{i}\right), e^{x}\left(t_{k}^{i}\right), e^{v}\left(t_{k}^{i}\right)\right. \\
& -k \sum_{j=1}^{N}\left(L_{j, .}\left(t_{k}^{i}\right)+B_{j,}\left(t_{k}^{i}\right)\right) \varepsilon_{j}\left(t_{k}^{i}\right) \\
& -k r \sum_{j=1}^{N}\left(L_{j, .}\left(t_{k}^{i}\right)+B_{j,} \cdot\left(t_{k}^{i}\right)\right) \eta_{j}\left(t_{k}{ }^{i}\right) .
\end{aligned}
$$

Suppose $\sigma_{i}=t-t_{k}^{i}$, we have

$$
\left\|\eta_{i}\left(t_{k}{ }^{i}\right) \sigma_{i}+\varepsilon_{i}\left(t_{k}^{i}\right)\right\|^{2}+\left\|\pi \sigma_{i}+\eta_{i}\left(t_{k}^{i}\right)\right\|^{2} \leq \gamma \Omega \sigma_{i}^{2} .
$$

We obtain that $\sigma_{i}=0$, and the inequality is not true; $\sigma_{i}>0$ that is to say $t-t_{k}{ }^{i}>0$. To sum up, the self-triggering control strategy of the follower vehicle at $t_{k}^{i+1}$ moment is determined by the following conditions:

$$
\left\|\eta_{i}\left(t_{k}^{i}\right) \sigma_{i}+\varepsilon_{i}\left(t_{k}^{i}\right)\right\|^{2}+\left\|\pi \sigma_{i}+\eta_{i}\left(t_{k}^{i}\right)\right\|^{2}=\gamma \Omega \sigma_{i}^{2}
$$

If there is a $\sigma_{i}>0$ which satisfies (48), we get the next trigger time $t_{k}^{i+1}=\sigma_{i}+t_{k}^{i}$. In particular, if the topology of the vehicle queue changes at time $t$, so that $t_{k}^{i+1}=t$.

REMARK 3. The existence of $\sigma_{i}$ indicates that a Zeno behavior does not exist. At the same time, it indicates that the self-triggered control strategy can achieve the leader-follower consistency of vehicle formation under the circumstance that both the topology structure and the leader vehicle speed are time varying. The proof of stability is the same to the event-triggered control. It is thus avoided here.

\section{SIMULATION}

In this section, we will give two numerical experiments to verify the correctness and validity of the above theorems. Both experiments are based on a leader-follower vehicle formation system, which consist of a leader vehicle and four follower vehicles.

Firstly, we verify that the speed of the leader vehicle is constant. The dynamic equation of leader and follower are shown below:

$$
\left\{\begin{array} { l } 
{ \dot { x _ { 0 } } ( t ) = v _ { 0 } ( t ) , } \\
{ \dot { v _ { 0 } } ( t ) = 0 , }
\end{array} \quad \left\{\begin{array}{l}
\dot{x}_{i}(t)=v_{i}(t) \\
\dot{v}_{i}(t)=u_{i}(t)
\end{array}\right.\right.
$$

where $u_{i}(t)$ is defined in (5), $k=3.4, r=1.2$. and the parameters satisfy the conditions in Theorem 3.1.

In order to more intuitively verify the effectiveness of the selftriggering control strategy proposed in this paper, we assume that the vehicle formation system carries out three topology switches. The topology structure between vehicles at the initial moment is shown in Figure 2. Each adjacency matrix $A$ and coefficient 


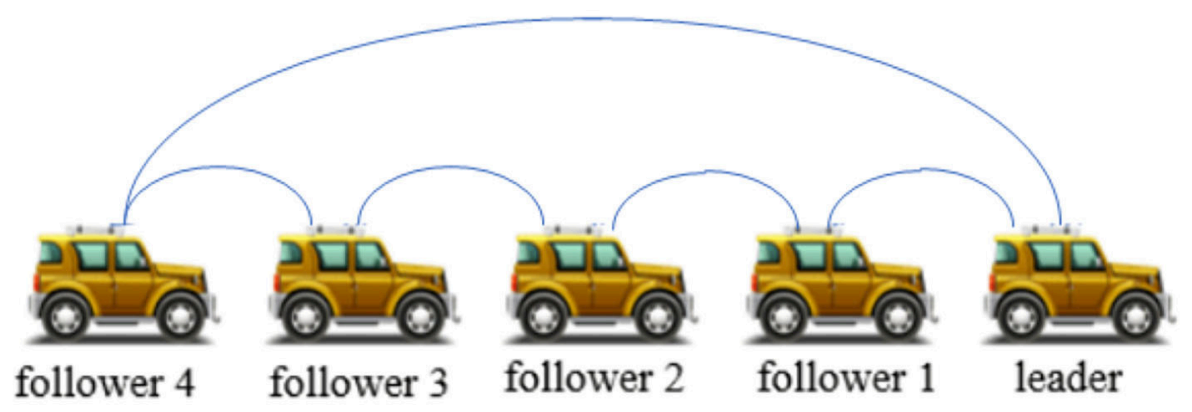

FIGURE 2 | Topology at initial time.

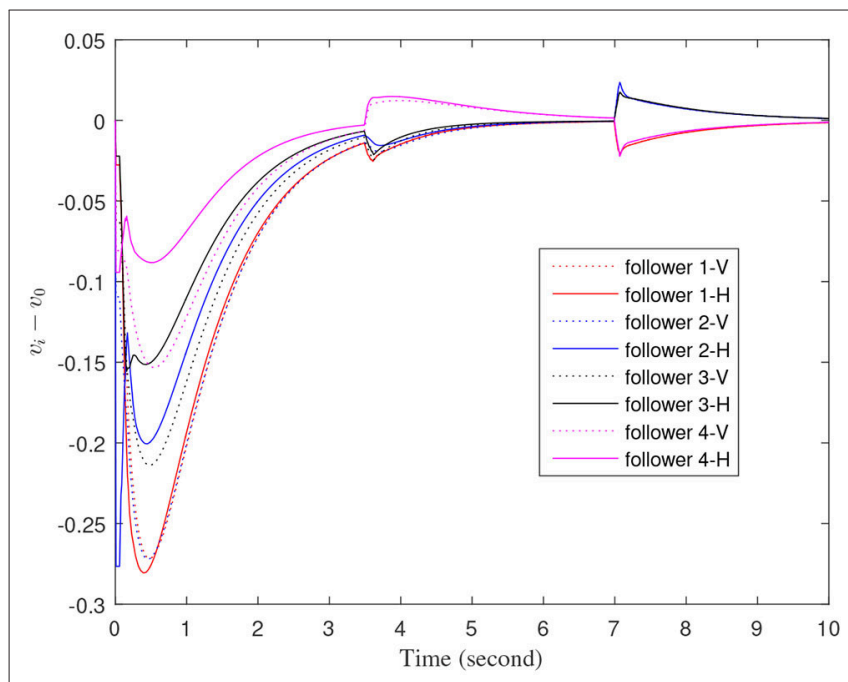

FIGURE 3 | The velocity error between the follower car and the leader car.

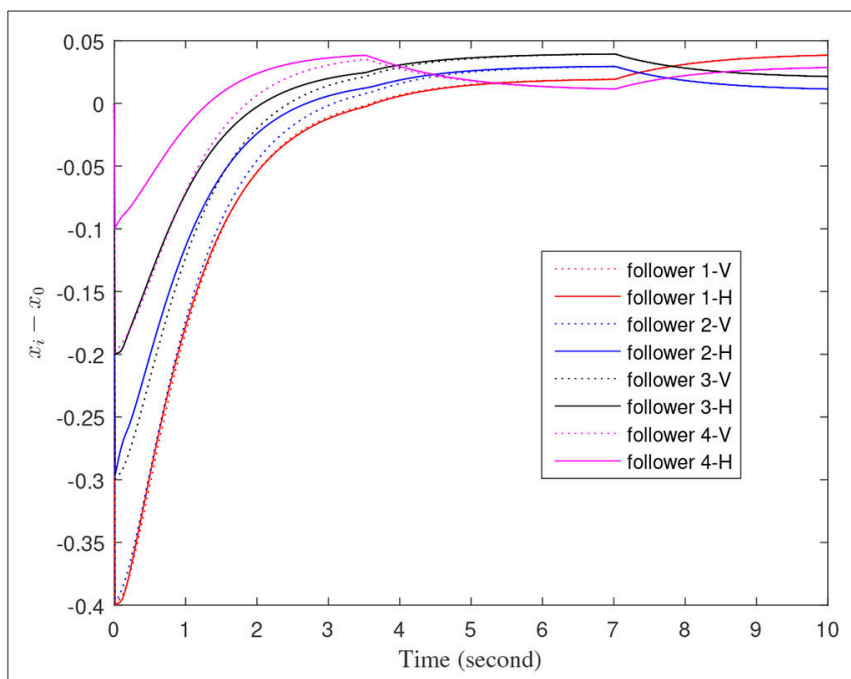

FIGURE 4 | The real-time distance between each follower car and the leader car.

The initial values of the leader vehicle and the follower vehicle are defined as follows:

$$
\begin{aligned}
& x_{0}(0)=(0,0), \quad x_{1}(0)=(-0.4,-0.5), \quad x_{2}(0)=(-0.3,-0.3), \\
& x_{3}(0)=(-0.2,-0.4), x_{4}(0)=(-0.2,-0.1), \\
& v_{0}(0)=(0.1,0.1), \quad v_{1}(0)=(0.15,0.1) \\
& v_{2}(0)=(0.1,0.12), \\
& v_{3}(0)=(0.15,0.1), \quad v_{4}(0)=(0.18,0.2) .
\end{aligned}
$$

Figures 3-7 are the results for the leader vehicle at constant speed. Figure 3 shows the velocity error between the follower car and the leader car. Figure 4 express as the real-time distance between each follower car and the leader car. Figure 5 express as the changes in the controller of each follower car. Because the topology is changed, the controller changed dramatically twice. The self-trigger interval of each follower are displayed in Figure 6. Figure 7 shows the relative position of vehicles when the formation is finally stabilized.

As we can see from Figures 3-5, when the topology changes, the controller of the follower vehicle adjusts the vehicle speed to keep the vehicle in formation and the error of vehicle speed 
tends to zero over time. This indicates that the controller can adapt to various topological switching situations by adjusting the control intensity. At the same time, due to the change of topology, the relative positions between vehicles will also change, and the follower vehicles will constantly adjust their positions to the new relative positions under the action of the controller. It is worth noting that the position error of the follower vehicles does not gradually approach zero as time goes on, and it reaches a fixed value greater than zero in Figure 4. This fixed value is the safe distance $\left(h_{i j} v_{0}\right)$ between the vehicles. As shown in Figure 7, when a stable state is reached, the vehicles should keep a safe distance from each other. Moreover the self-triggering instants are displayed in Figure 6. The simulation results exhibit that the controller and the self-triggering control strategy designed by us have a good performance. It achieves the stability of vehicle formation system under the condition of constant topological changes. Moreover, the vehicles can keep a safe distance.

Secondly, we verify that the speed of the leader vehicle is time varying. In order to more intuitively verify the effectiveness of the self-triggering control strategy, we randomly selected several

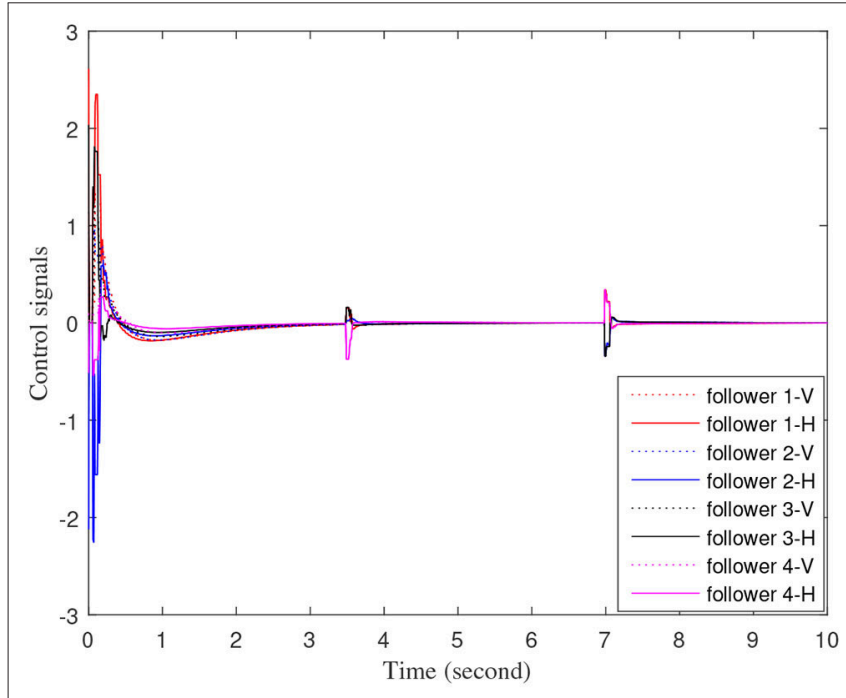

FIGURE 5 | Control input signals of each follower vehicle.

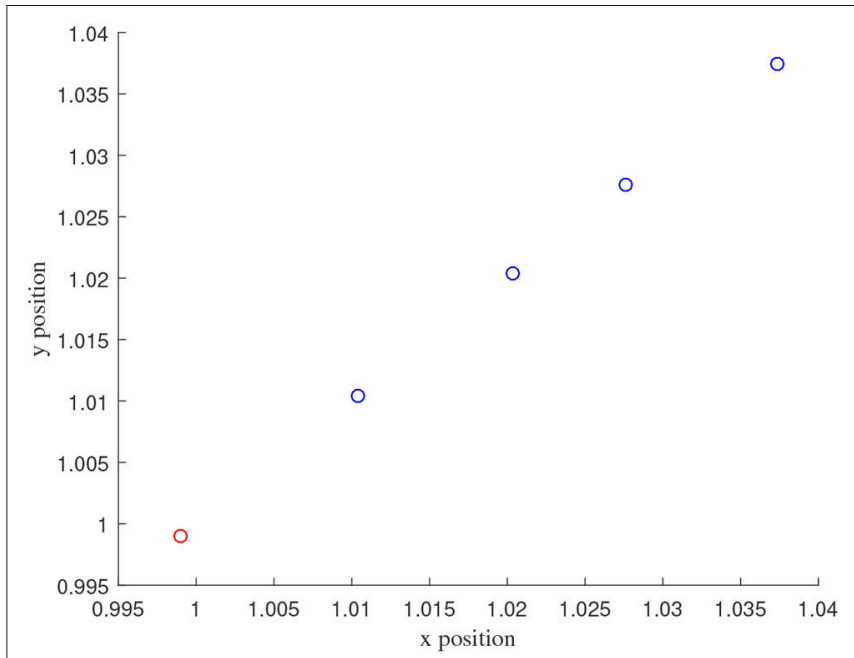

FIGURE 7 | The position information of the vehicle when the formation is stable.


FIGURE 6 | The event trigger interval of each follower vehicle. 


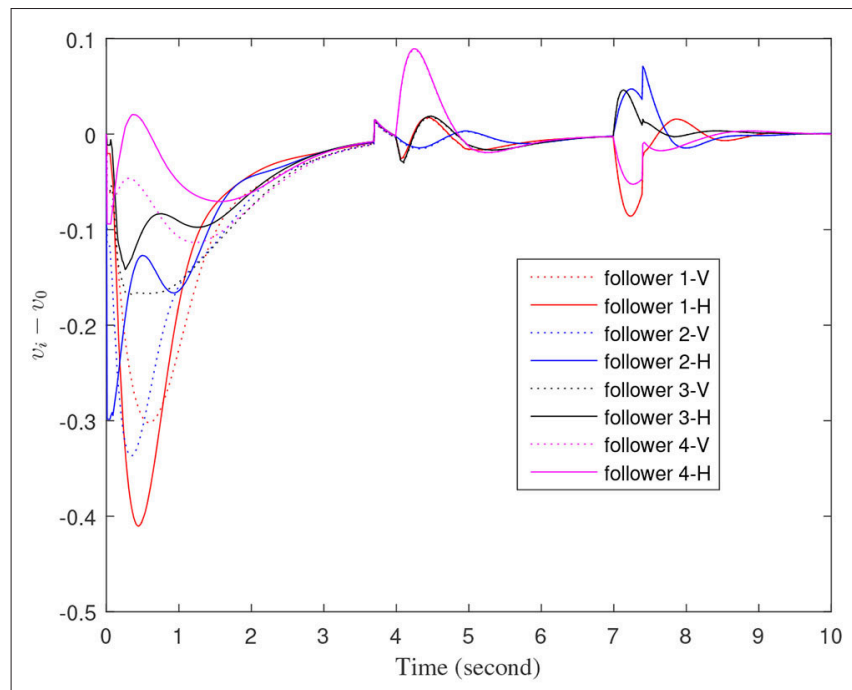

FIGURE 8 | The velocity error between the follower car and the leader car.

time points and used the leader vehicle dynamics Equation (4) to change the state of the leader vehicle. The dynamic equation of the leading vehicle are as follows

$$
\left\{\begin{array}{l}
\dot{x_{0}}(t)=\dot{v_{0}}(t) \\
\dot{v_{0}}(t)=-\sin \left(x_{0}(t)\right)-0.25 v_{0}(t)+1.5 \cos (2.5 t)
\end{array}\right.
$$

When the speed of leader changes, the dynamic equation of the follower's vehicle is defined as

$$
\left\{\begin{array}{l}
\dot{x}_{i}(t)=\dot{v}_{i}(t) \\
\dot{v}_{i}(t)=f\left(t, x_{i}, v_{i}\right)-f\left(t, x_{0}, v_{0}\right)+u_{i}(t)
\end{array}\right.
$$

where $f(t, x, v)=-\sin (x)-0.25 v+1.5 \cos (2.5 t)$.

The define of Figures 8-12 is similar to Figures 3-7, but Figures 8-12 show the results of a leader with time-varying velocity. From Figures 8-10, we can see that when the speed of the leader vehicle or topology changes, the follower vehicle can quickly adapt to the changing so that its speed is consistent with the leader vehicle, and the real-time distance between follower vehicle and leader vehicle change rapidly. Moreover, it can be seen from Figure 10, that after the vehicle formation system reaches stability, the controller of the follower vehicle no longer exerts control. The self-triggering instants are displayed in Figure 11. Notably, after the leader vehicle speed changes, the safety distance of the follower vehicle also changes in Figure 9. However, the vehicles ultimately kept a safe distance, as shown in Figure 12. The simulation results show that the controller and the self-triggering control strategy designed in this paper have a good performance. It can make the vehicle formation system reach stable state under the condition of changing topology and leader speed.

The number of triggers with a distributed event-triggered control scheme in Yang et al. (2018) and self-triggered control scheme (41) within $0-15 \mathrm{~s}$ are shown in the Table 1. What we can

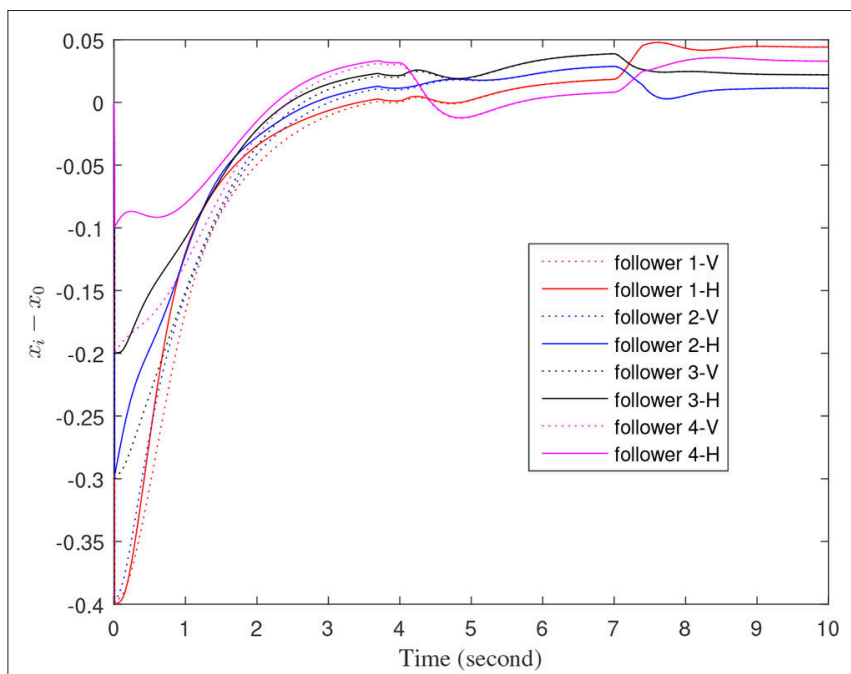

FIGURE 9 | The real-time distance between each follower car and the leader car.

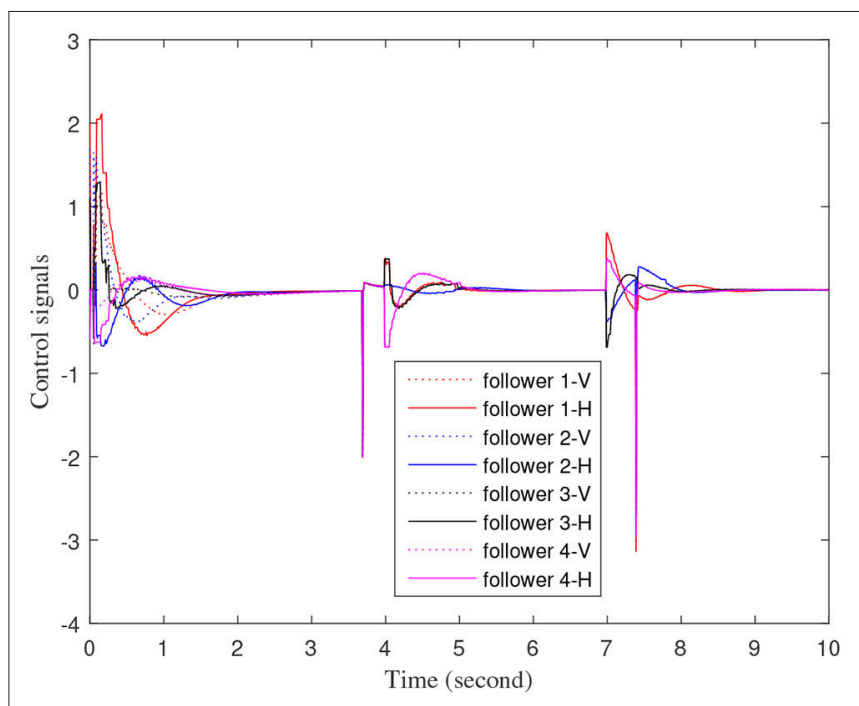

FIGURE 10 | Control input signals of each follower vehicle.

obtain from Table $\mathbf{1}$ is that the self-triggered control scheme (41) needs less triggering events than the distributed event-triggered control scheme in Yang et al. (2018). At the same time, the mean time interval which represents the average time between each trigger in Table 2 indicates that the self-triggered control strategy designed in this paper has a lower trigger probability and execution moment. It shows that the self-triggered control strategy proposed here can effectively reduce the energy loss of data detection and calculation in the control process.

\section{CONCLUSIONS}

In this paper, we have studied leader-follower consistency in vehicle formation systems with time-varying topology under 

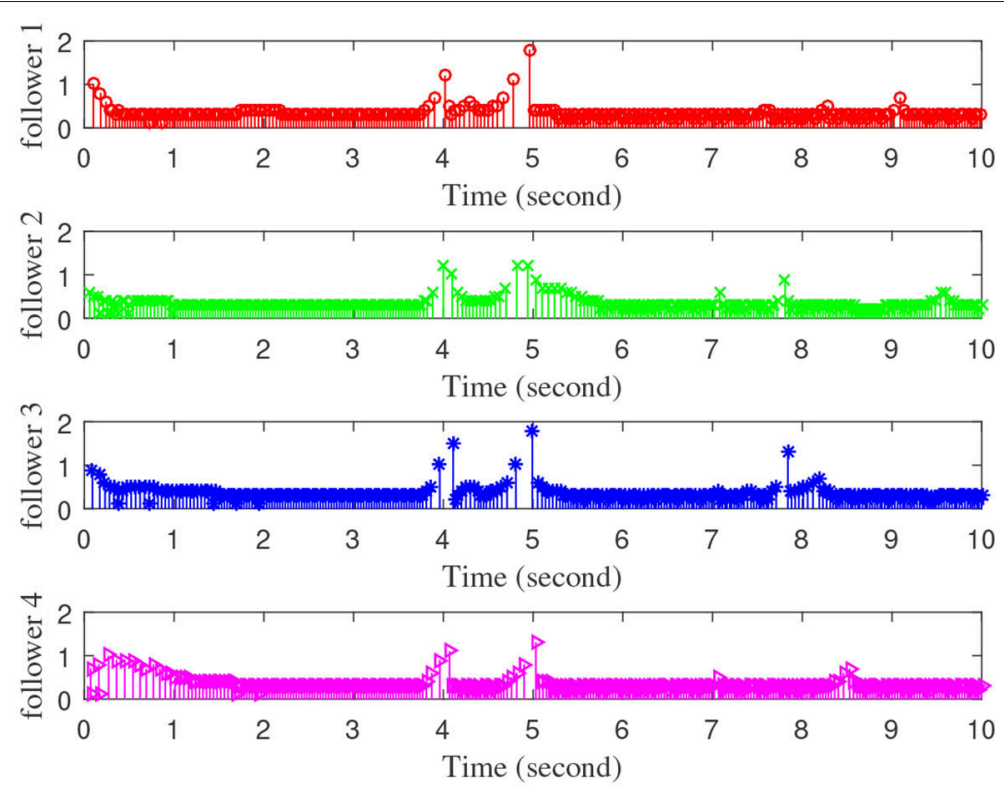

FIGURE 11 | The event trigger interval of each follower vehicle.

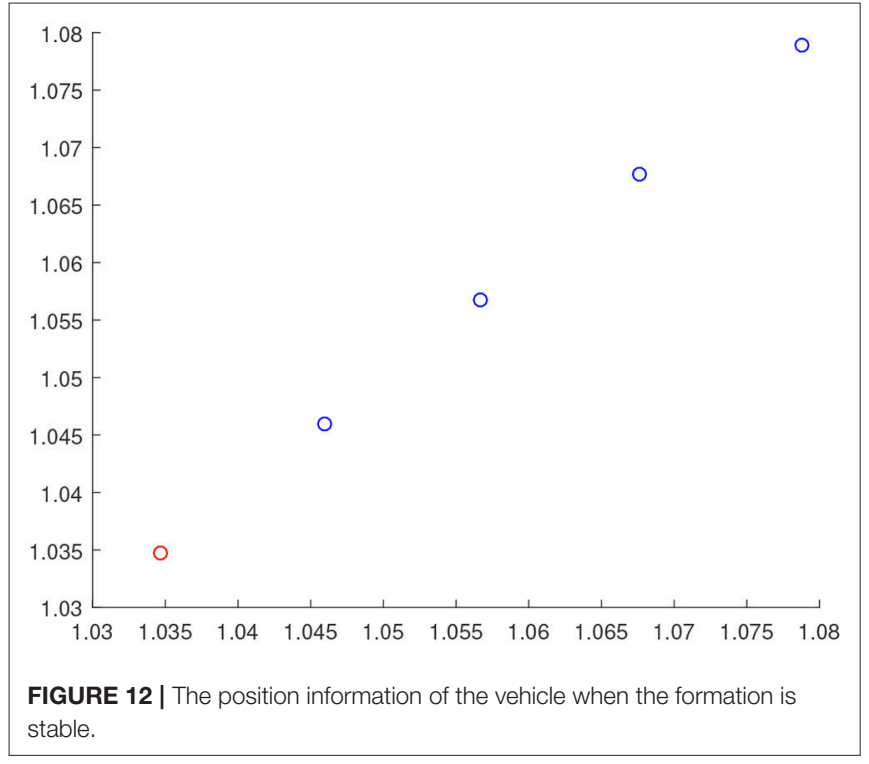

TABLE 1 | Triggered numbers of follower agents.

\begin{tabular}{lcccc}
\hline \multirow{2}{*}{ Control strategy } & \multicolumn{4}{c}{ Numbers of triggered events of agents } \\
\cline { 2 - 5 } & $\mathbf{1}$ & $\mathbf{2}$ & $\mathbf{3}$ & Total \\
\hline $\begin{array}{l}\text { Event-triggered in Yang } \\
\text { et al. (2018) }\end{array}$ & 582 & 857 & 1,029 & 2,468 \\
\begin{tabular}{l} 
Self-triggered (41) \\
\hline
\end{tabular} & 275 & 234 & 226 & 735 \\
\hline
\end{tabular}

event-triggering mechanism. The difference between our work and the published papers is that we have designed a selftriggering control strategy that avoids continuous calculation and
TABLE 2 | Mean time interval of follower agents.

\begin{tabular}{lccc}
\hline \multirow{2}{*}{ Control strategy } & \multicolumn{3}{c}{ Mean time interval } \\
\cline { 2 - 4 } & $\mathbf{1}$ & $\mathbf{2}$ & $\mathbf{3}$ \\
\hline Event-triggered in Yang et al. (2018) & 0.0127 & 0.0119 & 0.0151 \\
Self-triggered (41) & 0.0546 & 0.0641 & 0.0662
\end{tabular}

measurement and reduces the loss of communication resources. At the same time, we have proved the consistency of the system under the control of the trigger function. In addition, we have also studied the consistency of the vehicle formation system with time-varying topology when the leader speed is time varying. Finally, the effectiveness of the proposed controllers has been verified by numerical experiments. In addition, it should be noted that, although we proved the stability of formation system by Lyapunov function, we did not give its string stability which will be studied in the future.

\section{DATA AVAILABILITY STATEMENT}

All datasets generated for this study are included in the article/supplementary material.

\section{AUTHOR CONTRIBUTIONS}

All authors listed have made a substantial, direct and intellectual contribution to the work, and approved it for publication. 


\section{FUNDING}

This work was supported in part by the 2018 industrial Internet innovation and development project "construction of industrial Internet security standard system and test and verification environment", in part by the National Key Research and Development Program of China under Grant

\section{REFERENCES}

Chu, X., Peng, Z., Wen, G., and Rahmani, A. (2019). Distributed formation tracking of nonholonomic autonomous vehicles via eventtriggered and sampled-data method. Int. J. Control 92, 2243-2254. doi: 10.1080/00207179.2018.1436193

De Persis, C., and Frasca, P. (2013). Robust self-triggered coordination with ternary controllers. IEEE Trans. Automat. Control 58, 3024-3038. doi: 10.1109/TAC.2013.2273302

Dolk, V. S., Ploeg, J., and Heemels, W. M. H. (2017). Event-triggered control for string-stable vehicle platooning. IEEE Trans. Intell. Transport. Syst. 18, 3486-3500. doi: 10.1109/TITS.2017.2738446

Du, H., Zhou, J., Wu, D., and Wen, G. (2017). "Consensus for second-order nonlinear leader-following multi-agent systems via event-triggered control," in 2017 International Workshop on Complex Systems and Networks (IWCSN) (Hefei), 301-305. doi: 10.1109/IWCSN.2017.8276543

Fax, J. A., and Murray, R. M. (2004). Information flow and cooperative control of vehicle formations. IEEE Trans. Automat. Control 49, 1465-1476. doi: 10.1109/TAC.2004.834433

Fei, Z., Wang, X., Liu, M., and Yu, J. (2019). Reliable control for vehicle active suspension systems under event-triggered scheme with frequency range limitation. IEEE Trans. Syst. Man Cybernet. Syst. doi: 10.1109/TSMC.2019.2899942

Hee Lee, G., Faundorfer, F., and Pollefeys, M. (2013). "Motion estimation for self-driving cars with a generalized camera," in Proceedings of the IEEE Conference on Computer Vision and Pattern Recognition (Zurich), 2746-2753. doi: 10.1109/CVPR.2013.354

Lafferriere, G., Williams, A., Caughman, J., and Veerman, J. (2005). Decentralized control of vehicle formations. Syst. Control Lett. 54, 899-910. doi: 10.1016/j.sysconle.2005.02.004

Lantos, B., and Max, G. (2016). Hierarchical control of unmanned ground vehicle formations using multi-body approach. Acta Polytech. Hung. 13, 137-156. doi: 10.12700/APH.13.1.2016.1.10

Li, W., Zhang, G., Pan, T., Zhang, Z., Geng, Y., and Wang, J. (2019). A lipschitz optimization-based mppt algorithm for photovoltaic system under partial shading condition. 7, 126323-33

Li, Y., Tang, C., Li, K., Peeta, S., He, X., and Wang, Y. (2018). Nonlinear finite-time consensus-based connected vehicle platoon control under fixed and switching communication topologies. Transport. Res. C Emerg. Technol. 93, 525-543. doi: 10.1016/j.trc.2018.06.013

Li, Z., Hu, B., Li, M., and Luo, G. (2019). String stability analysis for vehicle platooning under unreliable communication links with eventtriggered strategy. IEEE Trans. Vehicular Technol. 68, 2152-2164. doi: 10.1109/TVT.2019.2891681

Munz, U., Papachristodoulou, A., and Allgower, F. (2011). Consensus in multiagent systems with coupling delays and switching topology. IEEE Trans. Automat. Control 56, 2976-2982. doi: 10.1109/TAC.2011.2161052

Peters, A. A., Mason, O., and Middleton, R. H. (2016). "Leader following with non-homogeneous weights for control of vehicle formations," in 2016 IEEE Conference on Control Applications (CCA) (Valparaiso: IEEE), 109-113. doi: 10.1109/CCA.2016.7587830
No. 2018YFB0803505, in part by the University of Science and Technology Beijing under Grant FRF-BD-19-012A and Grant FRF-TP-19-005A3, in part by the National Natural Science Foundation of China under Grant 81961138010, Grant U1736117 and Grant U1836106, and in part by the Technological Innovation Foundation of Shunde Graduate School, USTB, under Grant BK19BF006.

Ren, W. (2007). Multi-vehicle consensus with a time-varying reference state. Syst. Control Lett. 56, 474-483. doi: 10.1016/j.sysconle.2007.01.002

Saboori, I., and Khorasani, K. (2014). $H^{\infty}$ consensus achievement of multi-agent systems with directed and switching topology networks. IEEE Trans. Automat. Control 59, 3104-3109. doi: 10.1109/TAC.2014.2358071

Satur, O. R., and Kharchenko, N. V. (2020). A model of dynamical system for the attainment of consensus. Ukr. Math. J. 71, 1456-1469. doi: 10.1007/s11253-020-01725-w

Stojković, I., and Katić, D. (2017). Formation control of robotized aerial vehicles based on consensus-based algorithms. FME Trans. 45, 559-564. doi: $10.5937 / \mathrm{fmet} 1704559 \mathrm{~S}$

Viegas, D., Batista, P., Oliveira, P., and Silvestre, C. (2018). Discrete-time distributed Kalman filter design for formations of autonomous vehicles. Control Eng. Pract. 75, 55-68. doi: 10.1016/j.conengprac.2018.03.014

Vilarinho, C., Tavares, J. P., and Rossetti, R. J. (2016). Design of a multiagent system for real-time traffic control. IEEE Intell. Syst. 31, 68-80. doi: 10.1109/MIS.2016.66

Wang, J., Y. Xu, J. Z., and Yang, D. (2018). Time-varying formation for general linear multi-agent systems via distributed event-triggered control under switching topologies. Chin. Phys. B. 27:040504. doi: 10.1088/1674-1056/27/4/040504

Wang, Z., Wu, G., and Barth, M. J. (2017). Developing a distributed consensus-based cooperative adaptive cruise control system for heterogeneous vehicles with predecessor following topology. J. Adv. Transport. 2017:1023654 doi: $10.1155 / 2017 / 1023654$

Wei, Y., Liyuan, W., and Ge, G. (2017). Event-triggered platoon control of vehicles with time-varying delay and probabilistic faults. Mech. Syst. Signal Process. 87, 96-117. doi: 10.1016/j.ymssp.2016.09.042

Wen, S., Guo, G., Chen, B., and Gao, X. (2018). Event-triggered cooperative control of vehicle platoons in vehicular ad hoc networks. Inform. Sci. 459, 327-340. 341-353. doi: 10.1016/j.ins.2018.02.051

Yang, Y., Dong, Y., and Dou, C. (2018). Output-based event-triggered schemes on leader-following consensus of a class of multi-agent systems with lipschitz-type dynamics. Inform. Sci. 459, 327-340. doi: 10.1016/j.ins.2018. 02.020

Zhang, H., Zheng, X., Yan, H., Peng, C., Wang, Z., and Chen, Q. (2016). Codesign of event-triggered and distributed $H^{\infty}$ filtering for active semivehicle suspension systems. IEEE/ASME Trans. Mechatron. 22, 1047-1058. doi: 10.1109/TMECH.2016.2646722

Conflict of Interest: The authors declare that the research was conducted in the absence of any commercial or financial relationships that could be construed as a potential conflict of interest.

Copyright (C) 2020 Wang, Wang, Guo, Luo and Gao. This is an open-access article distributed under the terms of the Creative Commons Attribution License (CC BY). The use, distribution or reproduction in other forums is permitted, provided the original author(s) and the copyright owner(s) are credited and that the original publication in this journal is cited, in accordance with accepted academic practice. No use, distribution or reproduction is permitted which does not comply with these terms. 